\title{
Form discrimination and reversal learning in kittens'
}

\section{J. M. WARREN, The Pennsylvania State University, University Park, Pa. 16802}

Groups of nine kittens, aged 60,90, 120, and 180 days at the start of the experiment were tested on 10 reversals of a form discrimination task. The groups all showed significant improvement in reversal learning but did not differ significantly in performance at any stage in training. These negative results are similar to those previously obtained in a study of spatial reversal learning by kittens.

Cats aged 45 to 180 days do not differ significantly in performance on serial reversals of a discrimination with combined brightness and position cues (Warren \& Warren, 1967). The comparative literature suggests, however, that some vertebrates may learn spatial reversals with progressively fewer errors but fail to show any interreversal improvement when tested with visual cues (Bitterman, 1965). The present experiment investigated the possibility that performance on serial reversals of a nonspatial visual discrimination varies as a function of age in kittens.

\section{SUBJECTS}

The Ss were 36 kittens, 7 Siamese and 29 mongrels, divided into four groups of nine animals each which were $60,90,120$, or 180 days old at the start of the experiment.

The Ss were tested under low ( 0 - to 23-h) levels of food deprivation and reinforced for correct responses with small quantities of pork kidney or tuna fish.

\section{APPARATUS AND TRIAL PROCEDURE}

The discrimination apparatus was the modified Grice box, described by Warren \& Warren (1967) except the apparatus was medium grey throughout in this study. The discriminanda were a white vertically oriented circle and triangle, with areas of $81 \mathrm{~cm}$, cut from 1 -in. tumber. The objects were presented in fixed positions in the end compartments, approximately 5 in. from the rear wall of the response compartment. The incentive was placed immediately behind the correct stimulus and was invisible except from the side or rear.

A trial began when $\mathrm{E}$ lifted the guillotine door to the start box and ended when $S$ entered one of the response areas. If his choice was correct, he was allowed to approach and eat the incentive; if his choice was incorrect, he was allowed to approach the unrewarded stimulus but not to correct his error by going to the other stimulus.

\section{PROCEDURE \\ Adaptation}

Two days of adaptation training preceded testing on the first discrimination problem. No stimuli were presented on the first day of adaptation. S was given 20 trials with kidney or tuna presented in plain sight on both sides of the apparatus and simply required to eat the food in both compartments on every trial. On the second day of adaptation, the stimulus objects, a circle and a triangle, were presented on each of 20 trials with food being placed in front of both objects on Trials 1 through 10 , and concealed behind both objects on Trials 11 through 20. The position of the objects was varied from trial to trial in a balanced irregular sequence, and $E$ recorded $S$ 's initial responses to determine which stimulus was preferred under conditions of nondifferential reinforcement.

Object Discrimination and Reversal Training

Subjects were required to choose the stimulus which was not preferred in adaptation testing and trained to a criterion of 10 consecutive correct responses in a single session on the original discrimination and on 10 serial reversals of the same discrimination with the two objects alternately being correct and incorrect as successive criteria were attained.

Twenty trials were given each day unless $S$ reached criterion in fewer trials. The beginning of training on each new reversal was always deferred until the session following the one in which $S$ met criterion on the preceding reversal task. The position of the rewarded stimulus was varied according to a balanced irregular sequence, and the noncorrection method was used throughout the experiment.

\section{RESULTS}

Reversal learning curves for the four groups of Ss are presented in Fig. 1. It is obvious that the form discrimination was a difficult one for the cats, since the groups averaged roughly 120 errors to criterion in initial learning (Reversal 0) and reached an apparent asymptote of approximately 40 errors to criterion on Reversals 6 to 10. The shape of the reversal curves is, however, almost identical with those obtained from cats tested on repeated reversals with more easily discriminated stimuli.

It will be noted that the oldest Ss, the 180-day group, made consistently more errors than any of the younger groups and that the 60-day-old kittens were generally superior to the three older groups in reversal learning. Analysis of variance indicates that improvement in reversal learning was highly significant $(F=11.70$, with $4 / 288 \mathrm{df}$ ) but that the difference between age groups did not approach significance $(F=1.66)$.

\section{DISCUSSION}

The observation that proficiency in learning serial reversals of a difficult form-discrimination problem is independent of age over the range from 60 to 180 days in kittens corroborates the negative findings of Warren and Warren's study of spatial reversal learning by kittens, and it provides additional support for the argument (Warren, 1967) that simple reversal learning tasks offer little promise for identifying important changes in problem solving capacity in mammalian phylogeny or ontogeny.

\section{REFERENCES}

BITTERMAN, M. E. The evolution of intelligence. Scientific American, $1965,212,92-100$

WARREN, J. M. An assessment of the reversal index. Animal Behaviour, 1967, 15, 493-498.

WARREN, J. M., \& WARREN, H. B. Spatial reversal learning by kittens. Journal of Comparative \& Physiological Psychology, 1967, 63, 523-525.

NOTE

1. This research was supported by Grant M-04276 from the National Institute of Mental Health, U. S. Public Health Service.

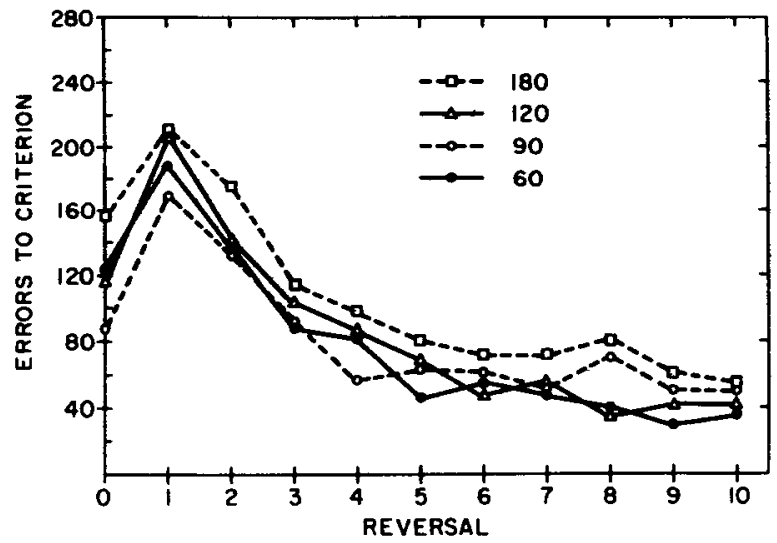

Fig. 1. Performance on serial reversals of a form discrimination by kittens as a function of age. 